\title{
Propiedades psicométricas de un instrumento para medir habilidades del pensamiento reflexivo en estudiantado universitario
}

\author{
Psychometrics Properties an Instrument for Gauge the Reflective Thinking in University Students \\ Propriedades psicométricas de um instrumento para medir habilidades do pensamento \\ reflexivo em estudantes universitários
}

\author{
Angélica Crespo-Cabuto \\ Instituto Tecnológico de Sonora \\ Sonora, México \\ angelica.crespo@itson.edu.mx \\ (iD) https://orcid.org/0000-0003-1846-2505 \\ María Teresa González-Frías \\ Instituto Tecnológico de Sonora \\ Sonora, México \\ mteresagf@hotmail.com \\ iD https://orcid.org/0000-0001-9085-0647 \\ Esthela Jacqueline Madrid-López \\ Instituto Tecnológico de Sonora \\ Sonora, México \\ esthelajacqueline.madridlopez@gmail.com \\ https://orcid.org/0000-0002-5499-3015
}

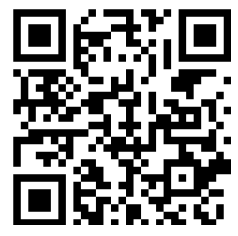

Recibido • Received • Recebido: 23 / 11 / 2017

Corregido • Revised • Revisado: 18 / 02 / 2019

Aceptado • Accepted • Aprovado: $10 / 05 / 2019$

Resumen: El estudio estableció las propiedades psicométricas de un instrumento para medir habilidades del pensamiento reflexivo en el desarrollo formativo de estudiantado universitario. En el estudio participaron 263 estudiantes de universidad: 220 ( $83.7 \%$ ) del sexo femenino $(M$ edad $=20$, $D E=1.5$ años) y 43 ( $16.3 \%$ ) del masculino ( $M$ edad $=20, D E=2.5$ años). Los resultados señalan que la escala es empíricamente sustentable para medir el contenido, confiabilidad y constructo mediante los factores de comprensión reflexiva, apoyo proceso reflexivo y manifestaciones del proceso reflexivo $\left(X^{2}\right.$ $\left.=20.08, g l=8,{ }^{*} p<.001 ; X^{2} / g l=1.397 ; C F I=.96 ; R M S E A=.039 ; G F I=.90\right)$. Se concluyó que el instrumento puede ser utilizado satisfactoriamente en la evaluación de las habilidades del pensamiento reflexivo en estudiantado universitario.

Palabras claves: Habilidades; pensamiento reflexivo; metacognición; validez. 
doi: http://dx.doi.org/10.15359/ree.23-3.16

URL: http://www.una.ac.cr/educare

CORREO: educare@una.cr

\begin{abstract}
The research determined psychometrics properties of an instrument to gage the reflective thinking in the formative development of university students. For this research 263 university students participated, of which $220(83.7 \%)$ were females $(M$ age $=20, D E=1.5$ years old), and 43 (16.3\%) were males ( $\mathrm{M}$ age $=20, \mathrm{DE}=2.5$ years old). The final result empirically supports scale to gage different factors, reflective compression, support reflective processes and demonstrations of reflective process $\left(X^{2}=20.08, g l=8,{ }^{*} p<.001 ; X^{2} / g l=1.397 ; C F I=.96 ; R M S E A=.039 ; G F I=.90\right)$. In conclusion, the instrument can be used on the evaluation of reflective thinking thought in university students.
\end{abstract}

Keywords: abilities, reflective thinking, metacognition, validity..

Resumo: $O$ estudo estabeleceu as propriedades psicométricas de um instrumento para medir habilidades do pensamento reflexivo no desenvolvimento formativo de estudantes universitários. No estudo participaram 263 estudantes universitários: 220 (83,7\%) mulheres ( $M$ idade=20, DE=1,5 anos) e $43(16,3 \%)$ homens ( $M$ idade $=20, D E=2,5$ anos). Os resultados indicam que a escala é empiricamente sustentável para medir conteúdo, confiabilidade e construção através dos fatores de compreensão reflexiva, suporte do processo reflexivo e manifestações do processo reflexivo $\left(X 2=20,08, g l=8,{ }^{*} p<.001\right.$; $\mathrm{X} 2 / \mathrm{gl}=1,397 ; \mathrm{CFI}=.96 ; \mathrm{RMSEA}=.039 ; \mathrm{GFI}=.90$ ). Concluiu-se que o instrumento pode ser utilizado satisfatoriamente na avaliação de habilidades de pensamento reflexivo em estudantes universitários.

Palavras-chave: Competências; pensamento reflexivo; metacognição; validade.

\title{
Introducción
}

En los ambientes de aprendizaje universitarios, la posibilidad de generar, desde el aula, las condiciones para la formación del pensamiento reflexivo implica construir procesos educativos con estrategias didácticas que faciliten la apropiación del conocimiento y, a su vez, estimulen el aprendizaje real en el estudiantado (Pereyra, 2008; Tishman, Perkins, y Jay, 1994). Este tipo de pensamiento se encuentra estrechamente ligado al concepto de "práctica reflexiva", por lo que las estrategias didácticas empleadas en el salón de clase deberán facilitar la práctica de lo aprendido en el aula, debido a que la capacidad de reflexión, junto con el pensamiento crítico y la resolución de problemas promueven una práctica informada y segura (Kuiper y Pezut, 2004).

Para la Universidad Australian Catholic University (2012), el pensamiento reflexivo se traduce en la capacidad de revisión de la experiencia y aprender de eso, especialmente cuando se trata de aplicar la teoría en la práctica, a través de dos tipos de análisis: "1) un análisis de las experiencias, perspectivas y decisiones de otros y 2) un análisis de las perspectivas personales que motivaron la toma de una decisión en el contexto de la práctica" (p. 2).

Mirzaei, Phang y Kahefi (2014) establecieron que las habilidades básicas de pensamiento reflexivo tienen impacto positivo en las habilidades profesionales de observación, comunicación, juicio, toma de decisiones y trabajo en equipo, por lo que si el profesorado utiliza estas 
habilidades durante sus procesos de enseñanza, puede desarrollar el pensamiento reflexivo del estudiantado, en todas las etapas del aprendizaje. En este mismo sentido, Calle (2013) estableció que es posible fortalecer este tipo de habilidades del pensamiento a partir de la implementación de estrategias didácticas, por parte del profesorado, encaminadas al desarrollo de la escritura de textos digitales.

Para identificar el desarrollo de este tipo de pensamiento en estudiantes, se han diseñado una serie de instrumentos de índole cualitativa y cuantitativa, pero no se cuenta con información suficiente sobre las propiedades psicométricas deestos. En el estudio realizado por Ossa-Cornejo, Palma-Luengo, Lagos-San Martín, Quintana-Abello y Díaz-Lorenas (2017), se analizaron los instrumentos para medir el pensamiento crítico del año 1980 al 2016, identificaron 97 estudios relacionados y que en los últimos 5 años se dio el mayor desarrollo de estos. Además se identificó que en América Latina existen pocos estudios de validez de instrumentos relacionados.

Llevar a cabo este tipo de ejercicios con estudiantes es de suma importancia, ya que en la actualidad les permite hacerse consientes de su formación integral, así como enfrentar los retos y solucionar los problemas de la sociedad del conocimiento, lo cual favorece el desarrollo ético en el ámbito personal y profesional (Cárdenas, Monroy, Arana y García, 2015).

Atendiendo la importancia de las habilidades del pensamiento reflexivo, en el estudio se propuso desarrollar un instrumento para medirlas, por lo que se determinaron las evidencias de validez de contenido, constructo y confiabilidad de una escala para medir las habilidades del pensamiento reflexivo en estudiantes de nivel superior, acorde a las características del contexto y del grupo de estudiantes que participó en la investigación, ya que existen pocos instrumentos validados en América Latina. Se partió de la hipótesis de que la escala cuenta con evidencias de validez y confiabilidad, lo cual le permite ser utilizada en la investigación de este constructo.

\section{Referentes conceptuales}

El pensamiento reflexivo se concibe como la actividad cognitiva que sustenta el actuar con cierto grado de originalidad, estableciendo una relación analítica entre la acción que se realiza y los fundamentos de una postura, por lo cual se requiere una operación intelectual, racional interna, mediante la cual se le presta atención a una serie de datos para dar sentido y ser combinados de una manera u otra (Romero, 2007; Sánchez, Santos y Ariza, 2005).

Desarrollar la capacidad de pensar sobre la acción se logra a través de la interacción constante con el ambiente natural, familiar y cotidiano del estudiantado mediante procesos de mediación con otros pares. El aprendizaje sucede por acciones de mediación a través del lenguaje, el cual funge como la herramienta que mediatiza las relaciones entre las personas, debido a la estrecha relación entre el pensamiento y lenguaje, entre la actividad mental y la palabra, por tanto, el habla y el pensamiento tienen una función esencial en la estructuración 
doi: http://dx.doi.org/10.15359/ree.23-3.16

URL: http://www.una.ac.cr/educare

CORREO: educare@una.cr

de la mente y la conciencia. Por ello, es necesario un ambiente de aprendizaje que promueva el pensamiento reflexivo, al crear redes de significado entre los recursos empleados para aprender y los resultados que el propio estudiantado identifica, donde el elemento metacognitivo favorece la formación de la autoconciencia y fortalece la función del uso de los recursos de pensamiento de manera explícita (León, 2014; Patiño, 2007).

Es así como el pensamiento reflexivo requiere de la mediación del lenguaje para hacerse explícito a través de tres funciones diferentes. En primer lugar se identifican los términos que marcan una postura epistémica, estos funcionan como indicadores de una actitud ante un conocimiento, son palabras que ayudan a caracterizar del pensamiento al hecho. En segundo lugar están los términos que describen un proceso intelectual, caracterizan el proceso de pensamiento y expresan su flujo, su estructura y su sentir, es decir, permiten discriminar las formas de pensar. Finalmente, están los términos que describen un producto intelectual, en este caso son sustantivos que nombran y marcan diferencias entre tipos de ideas, estas palabras son aquellas expresan un resultado (Perkins y Thisman, 2011).

Para analizar las habilidades del pensamiento reflexivo en el proceso de formación, es necesario considerar tres aspectos fundamentales, los cuales son la comprensión reflexiva, apoyos para el proceso reflexivo y la manifestación para el pensamiento reflexivo. En relación con la comprensión reflexiva, se entiende como un proceso que permite establecer el propio pensamiento a través del lenguaje, con la finalidad de lograr una transformación de intercambio social, por medio de una concientización individual sobre las propias acciones voluntarias y expresiones. Así mismo implica una forma de pensar rigurosa y eficiente para la aplicación de habilidades y herramientas que permitan prácticas perdurables en el estudiantado (GonzálezMoreno, 2012; Swartz, Costa, Beyer, Reagan y Kallick, 2013).

El segundo aspecto considera los apoyos para el proceso reflexivo, el cual es un eje principal en el proceso educativo, ya que tiene la finalidad de generar las condiciones para que el estudiantado se haga consciente respecto a lo que dice y hace. En este sentido, el personal docente debe brindar apoyo al estudiantado para el inicio de las actividades en el aula, con un andamiaje pertinente que le permita hacerse responsable y consciente de su propio aprendizaje. Para ello se debe considerar el lenguaje, el cual debe ser intencionado, ya que por medio de él se realizan intercambios sociales dentro y fuera del aula, así mismo es importante la motivación docente hacia el estudiantado, ya que debe valorar sus ideas y opiniones, con la finalidad de fomentar la participación e interés dentro en el aula (García-Rangel, García, y Reyes, 2014; González-Moreno, 2012).

En cuanto a las manifestaciones para el pensamiento reflexivo se reflejan en el proceso educativo, ya que este se convierte en un espacio de análisis de la realidad y permite avanzar hacia la construcción y transformación de la sociedad. Este proceso debe estar orientado para 
que el estudiantado logre claridad en tres aspectos fundamentales a) lo que hace, b) cómo se desarrollan sus propios procesos de aprendizaje; y 3 ) qué se esperaba de ellos, con la finalidad de que pueda ser consciente de su pensamientos y actos para que su desempeño profesional y personal favorezca al crecimiento social (González-Moreno, 2012; Sánchez et al., 2005).

\section{Método}

\section{Participantes}

Para la administración de los instrumentos fueron seleccionados, de manera no probabilística, 263 estudiantes de una universidad de un municipio del noroeste de México: 220 $(83.7 \%)$ del sexo femenino ( $M$ edad $=20, D E=1.5$ años) y $43(16.3 \%)$ del masculino $(M$ edad $=$ $20, D E=2.5$ años).

\section{Instrumentos}

Formación del pensamiento reflexivo. Se adaptó un instrumento considerando las categorías de análisis de pensamiento reflexivo propuestas por González-Moreno (2012), con un total de 23 reactivos, en donde se midió el constructo a través de las escalas de Comprensión reflexiva (ejemplo: Interpreto o comprendo lo que escucho (plática, conferencia, exposición, etc.), donde se evalúan las practicas que realiza el estudiante para desarrollar una comprensión reflexiva , Apoyos para el proceso reflexivo (ejemplo: Requiero de la motivación de mi profesor para desarrollar mis actividades académicas), que mide la búsqueda de apoyo para lograr un proceso reflexivo y Manifestaciones del pensamiento reflexivo (ejemplo: Considero diversas opciones para el desarrollo de las actividades académicas), que miden las practicas que desarrolla el estudiante al desarrollar una comprensión reflexiva.

Se contestó mediante una escala tipo Likert con cuatro opciones de respuesta que oscilaron desde 1 (Nunca), 2 (pocas veces), 3 (Casi siempre) y 4 (Siempre).

\section{Procedimiento}

Para realizar la validación del instrumento se llevó a cabo el diseño del instrumento a partir de las categorías del pensamiento reflexivo establecidas en la investigación de GonzálezMoreno (2012), y se establecieron los indicadores que permiten el logro en cada una de ellas; posteriormente se explicó, a las autoridades escolares, el objetivo del estudio y se solicitó su autorización para acceder a las aulas de los grupos de los tres programas educativos de una dirección académica; para lograr la participación del estudiantado, se realizó una invitación personal en las aulas, se le explicó el propósito del instrumento y se indicó la importancia de su apoyo y que se mantendría la confidencialidad de sus respuestas. 
doi: http://dx.doi.org/10.15359/ree.23-3.16

URL: http://www.una.ac.cr/educare

CORREO: educare@una.cr

Con la información recabada, se determinaron las propiedades psicométricas del instrumento, a partir de los análisis de evidencia sobre validez de contenido, mediante juicio de personal experto; validez de confiabilidad, la cual se estableció a partir el Alfa de Cronbach para identificar su consistencia interna, y a través de análisis de validez de constructo, para ello se desarrolló un análisis factorial exploratorio y confirmatorio.

\section{Resultados}

\section{Validez de contenido}

La escala se sometió a la validez de contenido mediante la revisión de cinco personas expertas en la temática de habilidades del pensamiento reflexivo con publicaciones en el área y con experiencia en psicometría, e interés por participar en el estudio (De Vellis, 2012; Sireci y Faulkner-Bond, 2014). Se les pidió que evaluaran cada ítems de la escala como adecuados o no adecuados para medir el constructo en la población a la que se dirigía el estudio. Debido a la concordancia en el grupo experto, se mantuvieron todos los ítems de la escala (Escobar-Pérez y Cuervo-Martínez, 2008).

\section{Confiabilidad por reactivo}

Se determinó la confiabilidad de los ítems mediante el estadístico Alfa de Cronbach, donde se decidió mantener todos los ítems que mostraran correlaciones superiores a .30 en relación con la escala (De Vellis, 2012). Por lo cual, se decidió no incluir un total de cuatro ítems de la escala original que afectaban la confiabilidad total de la escala, y resultó una escala de 19 ítems, con un valor de Alfa de .89 (Ver Tabla 1).

Tabla 1: Confiabilidad por Ítems de la Escala para medir la habilidades del pensamiento reflexivo

\begin{tabular}{|c|c|c|c|}
\hline Reactivos & $\begin{array}{c}\text { Correlación del } \\
\text { ítem-escala }\end{array}$ & $\begin{array}{c}\text { Alfa } \\
\text { eliminando el ítem }\end{array}$ & Decisión \\
\hline Aplico mis habilidades de expresión verbal (conversar, explicar, dialogar, etc.) & .22 & .89 & No incluirlo \\
\hline Emito información enriquecedora y que aporta valor a quienes me escuchan. & .52 & .85 & Incluirlo \\
\hline Tomo notas de explicaciones dadas, o de exposiciones de otras personas. & .23 & .89 & No incluirlo \\
\hline Interpreto o comprendo lo que escucho (plática, conferencia, exposición, etc.) & .55 & .85 & Incluirlo \\
\hline Soy consciente de lo que escribo al redactar evidencias de aprendizaje & .52 & .85 & Incluirlo \\
\hline Puedo realizar de manera voluntarias reflexiones sobre mi aprendizaje & .50 & .85 & Incluirlo \\
\hline Demuestro mi facilidad para comprender textos & .55 & .85 & Incluirlo \\
\hline
\end{tabular}


Relaciono la nueva información con información previamente adquirida

Establezco de manera correcta mis ideas en un texto

Muestro conciencia sobre los usos del lenguaje, como gramática, ortografía, puntuación y vocabulario.

Requiero de la motivación de mi docente para desarrollar mis actividades académicas Requiero del apoyo de algún compañero o compañer para lograr mi aprendizaje

Requiero ver ejemplos de evidencias de aprendizajes de mis compañeros o compañeras para identificar las propias

Puedo realizar mis actividades académicas de manera voluntaria para hacerme consciente de mis avances de aprendizaje

Tengo siempre claridad del objetivo de la construcción de mi aprendizaje en los cursos

Considero diversas opciones para el desarrollo de las actividades académicas

Utilizo diferentes estrategias para decidir las evidencias de aprendizaje que apoyan mi formación académica

Me hago preguntas de manera personal que orienten mi toma de decisiones en el desarrollo de mis actividades académicas

Las actividades académicas reflejan mi proceso reflexivo

Considero alguna experiencia similar escolar o personal para solucionar problemas

Identifico algún problema o dificultad a partir de esa experiencia; es decir, un obstáculo en la experiencia sobre el cual habría de trabajar.

Contrasto diferentes alternativas para dar solución a las dificultades identificadas

Pongo en práctica alguna de las alternativas de solución a las problemáticas o áreas de oportunidad identificadas

\begin{tabular}{|c|c|c|}
\hline .53 & .85 & Incluirlo \\
\hline .61 & .85 & Incluirlo \\
\hline 24 & .89 & No incluirlo \\
\hline .53 & .85 & Incluirlo \\
\hline .52 & .85 & Incluirlo \\
\hline .53 & .85 & Incluirlo \\
\hline .52 & .87 & Incluirlo \\
\hline .57 & .85 & Incluirlo \\
\hline .58 & .85 & Incluirlo \\
\hline .56 & .85 & Incluirlo \\
\hline .47 & .85 & Incluirlo \\
\hline .50 & .85 & Incluirlo \\
\hline 49 & .86 & Incluirlo \\
\hline .28 & .89 & No incluirlo \\
\hline .55 & .85 & Incluirlo \\
\hline .59 & .85 & Incluirlo \\
\hline
\end{tabular}

Nota: Elaboración propia.

\section{Validez de constructo}

Análisis factorial exploratorio. Para realizar este análisis se consideró lo establecido por De Vellis (2012), donde se indica que los ítems con carga factorial menor a .30, así como los que tienen cargas mayores a este valor. Se aplicó el método factorial de ejes principales, así como el de rotación Oblimin.

Los valores del KMO de .90 y de la prueba de esfericidad de Bartlett $\left(X^{2}=1625.88, p<.000\right)$ sugieren la adecuación de los datos para en el análisis. Se obtuvo una solución de tres factores que explican el $42.06 \%$ de la varianza de los puntajes. La correlación entre los factores sostiene la idea de que ambos miden el mismo constructo, en este caso el manejo de conflicto en padres y madres (ver Tabla 2). 
doi: http://dx.doi.org/10.15359/ree.23-3.16

URL: http://www.una.ac.cr/educare

CORREO: educare@una.cr

Tabla 2: Resultados del análisis factorial exploratorio de la Escala para medir las habilidades del pensamiento reflexivo

\begin{tabular}{|c|c|c|c|c|c|c|}
\hline \multirow[b]{2}{*}{ Ítem } & \multicolumn{6}{|c|}{ Carga Factorial } \\
\hline & M & $D E$ & F1 & $\mathrm{F} 2$ & F3 & $h^{2}$ \\
\hline Demuestro mi facilidad para comprender textos & 2.89 & .78 & .83 & .09 & .11 & .59 \\
\hline Relaciono la nueva información con información previamente adquirida & 3.09 & .72 & .68 & .04 & .05 & .41 \\
\hline Puedo realizar de manera voluntarias reflexiones sobre mi aprendizaje & 3.24 & .71 & .64 & .02 & .03 & .39 \\
\hline Establezco de manera correcta mis ideas en un texto & 3.01 & .71 & .59 & .05 & .13 & .47 \\
\hline Interpreto o comprendo lo que escucho (plática, conferencia, exposición, etc.) & 3.19 & 69 & .54 & .02 & .11 & .40 \\
\hline Emito información enriquecedora y que aporta valor a los que me escuchan. & 2.83 & .76 & .44 & .08 & .11 & .34 \\
\hline $\begin{array}{l}\text { Puedo realizar mis actividades académicas de manera voluntaria para hacerme consciente de mis } \\
\text { avances de aprendizaje }\end{array}$ & 3.12 & .79 & .42 & .04 & .23 & .37 \\
\hline $\begin{array}{l}\text { Pongo en práctica alguna de las alternativas de solución a las problemáticas o áreas de } \\
\text { oportunidad identificadas }\end{array}$ & 3.26 & .67 & .38 & .02 & .32 & .43 \\
\hline Contrasto diferentes alternativas para dar solución a las dificultades identificadas & 3.18 & .62 & .38 & .12 & .30 & .42 \\
\hline Requiero del apoyo de algún compañero o compañera para lograr mi aprendizaje & 2.14 & .80 & .00 & .81 & .08 & .66 \\
\hline $\begin{array}{l}\text { Requiero ver ejemplos de evidencias de aprendizajes de mis compañeros o compañeras para } \\
\text { identificar las propias }\end{array}$ & 2.35 & .83 & .01 & .78 & .09 & .62 \\
\hline Requiero de la motivación de mi docente para desarrollar mis actividades académicas & 2.52 & .86 & .01 & .49 & .12 & .32 \\
\hline Considero diversas opciones para el desarrollo de las actividades académicas & 3.11 & .68 & .06 & .03 & .77 & .54 \\
\hline $\begin{array}{l}\text { Utilizo diferentes estrategias para decidir las evidencias de aprendizaje que apoyan mi formación } \\
\text { académica }\end{array}$ & 3.01 & .72 & .01 & .10 & .67 & .45 \\
\hline $\begin{array}{l}\text { Me hago preguntas de manera personal que orienten mi toma de decisiones en el desarrollo de } \\
\text { mis actividades académicas }\end{array}$ & 3.13 & .81 & .01 & .04 & .58 & .35 \\
\hline Tengo siempre claridad del objetivo de la construcción de mi aprendizaje en los cursos & 3.13 & 67 & .12 & .03 & .56 & .46 \\
\hline Las actividades académicas reflejan mi proceso reflexivo & 3.09 & .67 & .20 & .04 & .39 & .35 \\
\hline Considero alguna experiencia similar escolar o personal para solucionar problemas & 3.07 & .76 & .19 & .06 & .36 & .35 \\
\hline Soy consciente de lo que escribo al redactar evidencias de aprendizaje & 3.34 & .64 & .25 & .05 & .35 & .34 \\
\hline \multicolumn{7}{|l|}{ Correlaciones entre los factores } \\
\hline Comprensión reflexiva & & & - & & & \\
\hline Apoyo proceso reflexivo & & & .40 & & & - \\
\hline Manifestaciones del proceso reflexivo & & & .71 & & & .41 \\
\hline
\end{tabular}

Nota: Elaboración propia. 
Análisis factorial confirmatorio. Para la realización del análisis factorial confirmatorio se empleó el método de estimación de máxima verosimilitud para determinar la bondad de ajuste empírica del modelo (Byrne, 2010; Cea, 2004) (Ver Tabla 3).

Tabla 3: Solución estandarizada por el análisis factorial confirmatorio para un modelo de tres factores

\begin{tabular}{|c|c|c|c|}
\hline \multirow[b]{2}{*}{ Ítem } & \multicolumn{3}{|c|}{ Factores } \\
\hline & $\begin{array}{l}\text { Comprensión } \\
\text { reflexiva }\end{array}$ & $\begin{array}{l}\text { Apoyo proceso } \\
\text { reflexivo }\end{array}$ & $\begin{array}{l}\text { Manifestaciones del } \\
\text { proceso reflexivo }\end{array}$ \\
\hline Demuestro mi facilidad para comprender textos & $.53^{* *}$ & & \\
\hline Relaciono la nueva información con información previamente adquirida & $.64^{* *}$ & & \\
\hline Puedo realizar de manera voluntarias reflexiones sobre mi aprendizaje & $.64^{* *}$ & & \\
\hline Establezco de manera correcta mis ideas en un texto & $.69^{* *}$ & & \\
\hline Interpreto o comprendo lo que escucho (plática, conferencia, exposición, etc.) & $.62^{* *}$ & & \\
\hline Emito información enriquecedora y que aporta valor a los que me escuchan. & $.69^{* *}$ & & \\
\hline $\begin{array}{l}\text { Puedo realizar mis actividades académicas de manera voluntaria para hacerme } \\
\text { consciente de mis avances de aprendizaje }\end{array}$ & $.60^{* *}$ & & \\
\hline $\begin{array}{l}\text { Pongo en práctica alguna de las alternativas de solución a las problemáticas o áreas } \\
\text { de oportunidad identificadas }\end{array}$ & $.67^{* *}$ & & \\
\hline Contrasto diferentes alternativas para dar solución a las dificultades identificadas & $.45^{* *}$ & & \\
\hline Requiero del apoyo de algún compañero o compañera para lograr mi aprendizaje & & $.47^{* *}$ & \\
\hline $\begin{array}{l}\text { Requiero ver ejemplos de evidencias de aprendizajes de mis compañeros o } \\
\text { compañeras para identificar las propias }\end{array}$ & & $.77^{* *}$ & \\
\hline Requiero de la motivación de mi docente para desarrollar mis actividades académicas & & $.74^{* *}$ & \\
\hline Considero diversas opciones para el desarrollo de las actividades académicas & & & $.55^{* *}$ \\
\hline $\begin{array}{l}\text { Utilizo diferentes estrategias para decidir las evidencias de aprendizaje que apoyan } \\
\text { mi formación académica }\end{array}$ & & & $67^{* *}$ \\
\hline $\begin{array}{l}\text { Me hago preguntas de manera personal que orienten mi toma de decisiones en el } \\
\text { desarrollo de mis actividades académicas }\end{array}$ & & & $.71^{* *}$ \\
\hline Tengo siempre claridad del objetivo de la construcción de mi aprendizaje en los cursos & & & $.65^{* *}$ \\
\hline Las actividades académicas reflejan mi proceso reflexivo & & & $.66^{* *}$ \\
\hline Considero alguna experiencia similar escolar o personal para solucionar problemas & & & $.68^{* *}$ \\
\hline Soy consciente de lo que escribo al redactar evidencias de aprendizaje & & & $.62^{* *}$ \\
\hline
\end{tabular}

${ }^{*} p<.05,{ }^{* *} p<.001$

Nota: Elaboración propia. 
doi: http://dx.doi.org/10.15359/ree.23-3.16

URL: http://www.una.ac.cr/educare

CORREO: educare@una.cr

Además, se consideraron los índices de ajuste, los cuales permiten confirmar lo establecido de manera empírica en el modelo (Blunch, 2013; Byrne, 2010; Cea, 2004) (Ver Tabla 4).

Tabla 4: Índices de ajuste del modelo para medir las habilidades del pensamiento reflexivo

\begin{tabular}{lcccccc}
\hline Modelo & $X^{2}$ & $P$ & $X^{2} / g l$ & CFI & RMSEA & GFI \\
\hline Tres Factores & 20.08 & $.001^{* * *}$ & 1.397 & .96 & .039 & .92 \\
\hline
\end{tabular}

$C F I=$ Índice de ajuste comparativo; RMSEA=Error de la raíz cuadrada media de aproximación; $G F I=$ Índice de bondad de ajuste

${ }^{*} p<.05 .{ }^{* *} p<.01 .{ }^{* *} p<.001$.

Nota: Elaboración propia.

\section{Discusión}

Los resultados confirman la hipótesis de que la escala cuenta con evidencias de validez y confiabilidad que justifican su utilización en la investigación acerca de las habilidades del proceso reflexivo en estudiantado universitario, determinan que estas pueden analizarse a partir de tres dimensiones: a) comprensión reflexiva, b) apoyos para el proceso reflexivo; y c) manifestaciones del pensamiento reflexivo. Sin embargo, la factibilidad de este estudio puede variar, si este se aplica en otros niveles educativos o con una población de un solo género.

El valor de la escala se refuerza por la importancia de realizar este tipo de análisis en estudiantado universitario, ya que las habilidades del pensamiento reflexivo posibilitan su autoconocimiento en los procesos de aprendizaje, lo cual impacta de manera positiva en el desarrollo del lenguaje, así como en su desempeño personal y profesional, permitiéndole hacer uso de comportamientos y procedimientos relevantes y significativos de manera pertinente, contribuyendo, así, al desarrollo exitoso del pensamiento (González-Moreno, 2012; Swartz et al, 2013).

Estudios como el realizado por Olivares y Heredia (2012) indican que es posible analizar el desarrollo del pensamiento reflexivo en estudiantes a partir de intervenciones específicas, en este caso, la del aprendizaje basado en problema, ya que se encontraron diferencias significativas entre los tres programas en los cuales se evaluaron a estudiantes que no desarrollaron aprendizaje con dicha estrategia y los que sí. Esto también coincide con el estudio realizado por Fullana, Pallisera, Palaudàrias y Badosa (2014), quienes, a partir de una entrevista, identificaron la percepción que tiene el estudiantado sobre las estrategias de aprendizaje que se utilizan para desarrollar el pensamiento reflexivo, la cual resulto en general positiva, ya que exploraron habilidades, emociones y comportamientos que favorecieron sus relaciones personales y profesionales. 
Otro aspecto importante a considerar es que este tipo de pensamiento se puede desarrollar siempre y cuando el profesorado aplique las habilidades en su propio aprendizaje, así como en la planeación de aprendizaje para otras personas, ya que esto le permite estar preparado para desarrollar pensamiento reflexivo en cualquier momento del proceso de aprendizaje del estudiantado (Mirzaei et al. (2014). Aunado a ello, Kuiper y Pesut (2004) mencionan que las estrategia para generar pensamiento crítico deben estar acompañadas de una estrategia metacognitiva, para lograr la autorregulación y reflexión del aprendizaje en el estudiantado.

\section{Conclusiones}

A partir del diseño del instrumento de medición de habilidades del pensamiento reflexivo en estudiantado universitario, se concluye que este cuenta con la validez de contenido, constructo y confiabilidad, por lo es pertinente su aplicación para identificar la percepción que tiene el estudiantado en relación con su logro en el proceso de formación, y se pueda tomar decisiones que lo favorezcan.

Se puede concluir que el desarrollo del pensamiento reflexivo en el estudiantado universitario es fundamental para que cuente con las competencias necesarias para su desarrollo profesional, sin embargo, esto requiere de una intervención guiada por parte del profesorado, a partir de estrategias de aprendizaje que lo favorezcan. Por lo tanto, el diseño de este instrumento permitirá identificar las áreas de oportunidad para docentes de la universidad; no obstante que los hallazgos sugieren que la escala es un instrumento valioso en la medición del constructo, es necesario destacar que esta posee ciertas limitaciones, ya que únicamente considera aspectos relativos a una autoevaluación del estudiantado sobre el proceso del pensamiento reflexivo. Esto lleva a sugerir a ampliar este instrumento con aspectos que evalúen la percepción del mismo personal docentes con respecto a las habilidades.

\section{Referencias}

Australian Catholic University. (2012). Thinking reflectively. Recuperado de https://leocontent. acu.edu.au/file/00de47c3-4f96-3ed6-c037-9498c917d4e3/7/Student\%20Skills\%20 Resource\%20Thinking\%20Reflectively-final.pdf

Blunch, N. J. (2013). Introduction to structural equation modeling using IBM SPSS statistics and

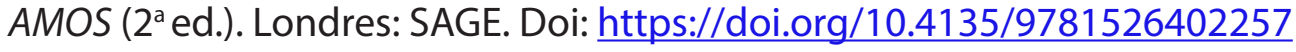

Byrne, B. (2010). Structural equation modeling with AMOS ( $2^{\mathrm{a}} \mathrm{ed}$.). New York: Routledge Taylor \& Francis Group.

Calle, G. Y. (2013). La evaluación de las habilidades del pensamiento crítico asociadas a la escritura digital. Revista virtual Universidad Católica del Norte, 40, 68-83. Recuperado de http://revistavirtual.ucn.edu.co/index.php/RevistaUCN/article/viewFile/446/938 
doi: http://dx.doi.org/10.15359/ree.23-3.16

URL: http://www.una.ac.cr/educare

CORREO: educare@una.cr

Cárdenas, L., Monroy, A., Arana, B. y García, M. (2015). Importancia del pensamiento reflexivo y crítico en enfermería. Revista mexicana de enfermería cardiológica, 23(1),35-41. Recuperado de www.medigraphic.com/pdfs/enfe/en-2015/en151f.pdf

Cea, M. (2004). Análisis multivariable. Teoría y práctica en la investigación social. Madrid: Síntesis.

De Vellis, R. (2012). Scale development. Theory and applications. New York: Sage.

Escobar-Pérez, J. y Cuervo-Martínez, Á. (2008). Validez de contenido y juicio de expertos: Una aproximación a su utilización. Avances en Medición, 6(1), 27-36. Recuperado de http:// www.humanas.unal.edu.co/psicometria/files/7113/8574/5708/Articulo3 Juicio de expertos 27-36.pdf

Fullana, J., Pallisera, M., Palaudàrias, J. y Badosa, M. (2014). El desarrollo personal y profesional mediante el aprendizaje reflexivo. Una experiencia en el grado de educación social. REDU: Revista de Docencia Universitaria, 12(1), 376-397. doi: https://doi.org/10.4995/ redu.2014.6413

García-Rangel, E. G., García, A. K. y Reyes, J. A. (2014). Relación maestro alumno y sus implicaciones en el aprendizaje. Ra Ximbai, 10(5), 279-290. Recuperado de https://www.redalyc.org/ pdf/461/46132134019.pdf

González-Moreno, C. X. (2012). Formación del pensamiento reflexivo en estudiantes universitarios. Magis: Revista Internacional de Investigación en Educación, 4(9), 595-617. Recuperado de https://www.redalyc.org/articulo.oa?id=281022848005

Kuiper, R. A. y Pesut, J. D. (2004). Promoting cognitive and metacognitive reflective reasoning skills in nursing practice: Self-regulated learning theory. Journal of Advanced Nursing, 45(4), 381-391. doi: https://doi.org/10.1046/j.1365-2648.2003.02921.x

León, F. R. (2014). Sobre el pensamiento reflexivo, también llamado pensamiento crítico. Propósitos y Representaciones, 2(1), 161-214. doi: http://dx.doi.org/10.20511/pyr2014. v2n1.56

Mirzaei, F., Phang, F. A. y Kashefi, H. (2014). Measuring teachers reflective thinking skills. Procedia - Social and Behavioral Sciences, 141, 640-647. doi: https://doi.org/10.1016/j. sbspro.2014.05.112

Olivares, S. L. y Heredia Y. (2012). Desarrollo del pensamiento crítico en ambientes de aprendizaje basado en problemas en estudiantes de educación superior. RMIE, Revista Mexicana de Investigación Educativa, 17(54), 759-778. Recuperado de http://www.scielo.org.mx/pdf/ rmie/v17n54/v17n54a4.pdf 
Ossa-Cornejo, C. J., Palma-Luengo, M. R., Lagos-San Martín, N. G., Quintana-Abello, I. M. y DíazLorenas, C. H. (2017). Análisis de instrumentos de medición del pensamiento crítico. Ciencias psicológicas, 11(1), 19-28. doi: http://dx.doi.org/10.22235/cp.v11i2.1343

Patiño, L. (2007). Aportes del enfoque histórico cultural para la enseñanza.Educación yEducadores, 10(1), 53-60. Recuperado de http://www.scielo.org.co/pdf/eded/v10n1/v10n1a05.pdf

Pereyra, M. (2008). Un aula para pensar (Cátedra de redacción). [Mensaje en un blo]. Recuperado de http://fcpolit.unr.edu.ar/blogs/redaccion1/2008/10/14/un-aula-para-pensar/

Perkins, D. y Tishman, S. (2011). El lenguaje del pensamiento. Educadores: Revista de renovación pedagógica, 236, 46-58.

Romero, L. (2007). Pensamiento reflexivo: Una aproximación inicial al ámbito de la formación de fonoaudiológos. Revista Chilena de Fonoaudiología, 8(1), 7-14. doi: https://doi. org/10.5354/0719-4692.2007.21373

Sánchez, S. I., Santos, M. T. y Ariza, M. V. (2005). Reflexionar para mejorar el acto educativo. Educación y Educadores, 8(1),145-159. Recuperado de http://www.redalyc.org/articulo. oa?id=83400811

Sireci, S. G. y Faulkner-Bond, M. (2014). Validity evidence based on test content. Psicothema, 26(1), 100-105.

Swartz, R. J., Costa, A. L., Beyer, B. K., Reagan, R. y Kallick, B. (2013). El aprendizaje basado en el pensamiento. Cómo desarrollar en los alumnos las competencias del siglo XXI. New York: Ediciones SM. Recuperado de http://ww2.educarchile.cl/UserFiles/P0001/File/robertswartz/libro-aprendizaje-basado-en-el-pensamiento-cap1.pdf

Tishman, S., Perkins, D. y Jay, E. (1994). Un aula para pensar. Aprender y enseñar en una cultura de pensamiento. Buenos Aires: Aiqu. 